\title{
Periodontal Assessment of Permanent Molar Teeth Restored with Stainless Steel Crown in Terms of Pocket Depth, Bleeding on Probing, Gingival Color and Inflammation
}

\author{
Alireza Heidari ${ }^{1}$, Mehdi Shahrabi ${ }^{2}$, Zahra Hosseini ${ }^{3}$, Nayereh M Sari ${ }^{4}$
}

\begin{abstract}
Aim: This study assessed the gingival health of permanent first molars before and 6 months after restoration with stainless steel crowns (SSCs). Materials and methods: This study was conducted on 23 children aged 6 to 12 years who required SSCs for their permanent first molars in one quadrant and had a contralateral first molar without SSC. Gingival color, inflammation, bleeding on probing (BOP), and probing pocket depth (PPD) were measured around both teeth. SSC was placed and, 6 months later, the same parameters were measured at both sides.

Results: Gingival color significantly improved and PPD, BOP, and inflammation in mesiobuccal and mesiolingual areas around the first molars restored with SSC significantly decreased after 6 months $(p<0.05)$. However, in distobuccal and distolingual areas, no significant change occurred in these indices 6 months after placement of SSC ( $p>0.05)$.

Conclusion: The results showed that SSCs with proper fit have no adverse effect on gingiva of permanent first molars given that the patient maintains a good oral hygiene.

Keywords: Crown, Gingiva, Molar, Permanent first, Stainless steel.

International Journal of Clinical Pediatric Dentistry (2019): 10.5005/jp-journals-10005-1607
\end{abstract}

\section{INTRODUCTION}

Chrome steel crowns were introduced by Humphrey in 1950 for use in children and adolescents. Stainless steel crowns (SSCs) are currently used for the restoration of primary or young permanent teeth with extensive caries, hypoplastic lesions or fracture, or as an attachment for appliances and space maintainers. ${ }^{1}$ Randel reviewed several studies on SSCs and found that SSCs are more durable and have a longer survival than multisurface amalgam restorations. ${ }^{2}$

The eruption of permanent first molars occurs in a period of life of children when they have a relatively high consumption of sugary substances and have a relatively poor oral hygiene. Also, most parents think that they are deciduous teeth. Thus, permanent first molars are susceptible to extensive caries. ${ }^{3}$

Gingival diseases may initiate during childhood and progress to periodontitis during the pubertal stage. Chronic marginal gingivitis is the most common gingival disease that occurs following extensive restorations in children. Dental plaque is the cause of gingivitis in both children and adults. Chronic marginal gingivitis often occurs as the result of placement of an ill-fitted crown and subgingival placement of margins, causing plaque accumulation at the site. ${ }^{3}$

Several previous studies have assessed periodontal status of teeth with SSCs or their adjacent teeth. Durr et al. in 1982 assessed plaque accumulation and gingival health around SSCs and concluded that the degree of inflammation was higher around teeth treated with an ill-fitted SSC compared to other teeth; whereas the gingival status of a tooth with a properly fitted crown was not significantly different from that of sound control teeth. However, no significant difference was noted between ill-fitted and ideal crowns or control teeth in terms of plaque accumulation. A correlation has been noted between plaque index and gingival index in teeth treated with ill-fitted SSCs. ${ }^{4}$ Einwag in 1984 found that
${ }^{1-4}$ Department of Pediatric Dentistry, Tehran University of Medical Science, Tehran, Iran

Corresponding Author: Zahra Hosseini, Department of Pediatric Dentistry, Tehran University of Medical Science, Tehran, Iran, Phone: +989128129604, e-mail: zahrahosseini69@gmail.com

How to cite this article: Heidari A, Shahrabi M, et al. Periodontal Assessment of Permanent Molar Teeth Restored with Stainless Steel Crown in Terms of Pocket Depth, Bleeding on Probing, Gingival Color and Inflammation. Int J Clin Pediatr Dent 2019;12(2):116-119.

Source of support: Nil

Conflict of interest: None

the use of precrimped crowns in primary molars caused clinically acceptable and insignificant gingival irritation in the primary and mixed dentition period; ${ }^{5}$ Guelmann et al. in 1998 found that welladapted SSCs on primary second molars had no significant effect on periodontal health of adjacent permanent first molars. ${ }^{6}$ Salama and Myers in 1992 evaluated SSCs in pediatric dentistry and stated that SSCs are suitable for primary teeth and also for temporary restoration of permanent molars and premolars. However, due to problems, SSCs are not recommended as a permanent crown for permanent teeth, which can be restored with amalgam. They showed that gingival status changes following placement of SSCs and they reported severe gingivitis in 33\%, moderate gingivitis in $25 \%$, and healthy gingiva in $13 \%$ of the cases. Myers found a significant association between the defective crown and gingivitis. ${ }^{7}$ Chao et al. in 1992 stated that SSCs are extensively used for primary and young permanent molars and a significant association exists between defective SSCs and periodontal problems. They assessed the effect of SSCs on gingival health and showed that ill-fitted

(c) The Author(s). 2019Open Access This article is distributed under the terms of the Creative Commons Attribution 4.0 International License (https://creativecommons. org/licenses/by-nc/4.0/), which permits unrestricted use, distribution, and non-commercial reproduction in any medium, provided you give appropriate credit to the original author(s) and the source, provide a link to the Creative Commons license, and indicate if changes were made. The Creative Commons Public Domain Dedication waiver (http://creativecommons.org/publicdomain/zero/1.0/) applies to the data made available in this article, unless otherwise stated. 
crowns caused deterioration of gingival index. ${ }^{8}$ Sharaf and Farsi in 2004 clinically and radiographically evaluated primary molars restored with SSCs and found no significant association between interproximal bone loss and extension of crown margins, marginal fit of SSCs, proper proximal contact between molars, oral hygiene status, or duration of presence of crown in the oral cavity; however, oral hygiene index of children significantly affected their gingival index. ${ }^{9}$ McLean et al. in 2007 evaluated anterior teeth restored with preveneered SSCs in terms of margin, adaptation, and size and concluded that NuSmile anterior preveneered SSCs are a clinically successful restoration for primary anterior teeth with early childhood caries. ${ }^{10}$ This study aimed to assess the periodontal status of permanent first molars at the baseline and 6 months after placement of SSCs.

\section{Materials and Methods}

This prospective study was conducted on 6-12-year-old children presenting to the Department of Pediatric Dentistry, Tehran University of Medical Sciences, School of Dentistry in 2015 who had one permanent first molar requiring SSC on one side and a sound permanent first molar or with a restoration other than SSC on the contralateral side. A total of 23 children were selected using convenience sampling. Demographic information of patients was collected and they were clinically examined on a dental chair. Patients with systemic diseases or mental retardation were excluded. Prior to any intervention, written informed consent was obtained from parents and the patients underwent clinical examination using a dental mirror, periodontal probe, and a dental explorer under adequate lighting. The permanent first molar tooth requiring SSC and the contralateral first molar (as control) were examined. Patients' age was recorded and their oral hygiene and saliva $\mathrm{pH}$ were assessed. The following parameters were examined:

- Color and inflammation of gingiva: gingiva was dried with a cotton roll and gauze prior to color determination. Normal gingiva is coral pink in color while inflamed gingiva is purplish red. For gingival color assessment, coral pink was allocated a score of 0 , red was allocated a score of 1 , and white was allocated a score of 2 .

- Probing pocket depth (PPD): it was measured in mesiobuccal, distobuccal, mesiolingual, and distolingual areas. The periodontal probe was inserted into the gingival sulcus parallel to the longitudinal axis of the tooth and was moved in a walking fashion.

- BOP: the periodontal probe was inserted into the gingival sulcus. Bleeding occurred in inflamed gingiva with atrophic or erosive pocket epithelium. The grading system used for BOP was as follows:

Grade 0: no bleeding

Grade 1: pin-point bleeding within 20 to 30 seconds

Grade 2: linear bleeding within 20 to 30 seconds covering the gingival sulcus

Grade 3: triangular BOP at the interdental area

Grade 4: bleeding immediately after probing in the interdental region and gingival sulcus around tooth. ${ }^{11}$

Next, SSC was placed on the respective tooth by a postgraduate student of pediatric dentistry. Necessary instructions were given to children and parents. After 6 months, the abovementioned parameters were measured again to assess the effect of SSC on gingival health. During this time period, no treatment was performed on the control teeth. Data were analyzed using SPSS via paired sample test, McNemar's test, and Wilcoxon signed rank test at 0.05 level of significance.

\section{Results}

Table 1 shows PPD in the test and control teeth at the baseline and after placement of SSC in four areas of mesiobuccal, distobuccal, mesiolingual, and distolingual. The data had a normal distribution. According to the paired sample $t$ test, the mean PPD in the mesiobuccal area of first molars before and after treatment with SSC had a significant difference $(p<0.05)$. Also, the mean PPD in the mesiobuccal area of control teeth showed a significant change after 6 months ( $p=0.02$ ). No significant difference was noted in PPD between the test and control teeth at the baseline $(p=1.0)$. Moreover, the mean PPD of test and control teeth was not significantly different at 6 months $(p=0.1)$.

According to the paired sample $t$ test, the mean PPD of test teeth was not significantly different at the baseline and 6 months after placement of SSC $(p=0.2)$. The mean PPD in the distobuccal area of control teeth did not significantly change after 6 months $(p=0.3)$. No significant difference was noted in the mean PPD of test and control teeth at the baseline $(p=0.1)$. The difference in this regard was not significant at 6 months either $(p=0.8)$. According to the paired sample $t$ test, the mean PPD in the mesiolingual area of test teeth significantly changed after 6 months $(p=0.04)$. However, this difference was not significant for the control teeth $(p=0.4)$. The mean PPD of test and control teeth was not significantly different

Table 1: Descriptive results of pocket depth in distolingual, mesiolingual, distobuccal, and mesiobuccal areas in 23 permanents first molars of 6-12-year-olds at the baseline and at 6 months after placement of SSC $(n=23)$

\begin{tabular}{|c|c|c|c|c|}
\hline & Minimum & Maximum & Mean & $\begin{array}{l}\text { Std. } \\
\text { deviation }\end{array}$ \\
\hline PPD, MB, SSC & 1 & 5 & 2.58 & 0.725 \\
\hline PPD, DB, SSC & 1 & 4 & 2.41 & 0.787 \\
\hline PPD, ML, SSC & 1 & 3 & 2.20 & 0.748 \\
\hline PPD, DL, SSC & 1 & 4 & 2.32 & 0.880 \\
\hline $\begin{array}{l}\text { PPD, MB, SSC } \\
\text { at } 6 \text { months }\end{array}$ & 1 & 4 & 2.04 & 0.467 \\
\hline $\begin{array}{l}\text { PPD, DB, SSC } \\
\text { at } 6 \text { months }\end{array}$ & 1 & 4 & 2.26 & 0.633 \\
\hline $\begin{array}{l}\text { PPD, ML, SSC } \\
\text { at } 6 \text { months }\end{array}$ & 1 & 3 & 1.85 & 0.629 \\
\hline $\begin{array}{l}\text { PPD, DL, SSC } \\
\text { at } 6 \text { months }\end{array}$ & 1 & 4 & 2.09 & 0.775 \\
\hline PPD, MB, control & 2 & 4 & 2.58 & 0.568 \\
\hline PPD, DB, control & 1 & 4 & 2.19 & 0.851 \\
\hline PPD, ML, control & 1 & 3 & 2.28 & 0.671 \\
\hline PPD, DL, control & 1 & 4 & 2.16 & 0.812 \\
\hline $\begin{array}{l}\text { PPD, MB, control } \\
\text { at } 6 \text { months }\end{array}$ & 2 & 4 & 2.24 & 0.643 \\
\hline $\begin{array}{l}\text { PPD, DB, control } \\
\text { at } 6 \text { months }\end{array}$ & 1 & 3 & 2.07 & 0.686 \\
\hline $\begin{array}{l}\text { PPD, ML, control } \\
\text { at } 6 \text { months }\end{array}$ & 1 & 3 & 2.15 & 0.382 \\
\hline $\begin{array}{l}\text { PPD, DL, control } \\
\text { at } 6 \text { months }\end{array}$ & 1 & 3 & 1.97 & 0.633 \\
\hline
\end{tabular}


at the baseline $(p=0.2)$. This difference was not significant at 6 months either $(p=0.05)$.

According to the paired sample $t$ test, the mean PPD in the distolingual area did not change after 6 months in the test group $(p=0.1)$. This change in the control group was not significant either $(p=0.06)$. Moreover, the mean PPD of the test and control groups was not significantly different at the baseline $(p=0.09)$. This difference at 6 months was not significant either $(p=0.3)$.

According to the results of McNemar's test, the mean BOP in the test teeth significantly changed after treatment ( $p=0.02)$; but this change was not significant in the control group $(p=0.08)$. The mean BOP in the test and control groups was not significantly different at the baseline or at 6 months $(p=1.0)$.

According to the Wilcoxon signed rank test, gingival color significantly improved at 6 months in the test group $(p=0.03)$ but no significant change was noted in the control group $(p=0.9)$. The difference between the test and control teeth in gingival color was significant at the baseline $(p=0.02)$ but this difference was not significant at 6 months after treatment $(p=0.4)$.

According to the results of McNemar's test, the degree of inflammation in the test tooth significantly changed at 6 months after placement of SSC $(p<0.05)$. This change in the control group was also significant $(p<0.05)$. The difference between the test and control teeth in the degree of inflammation was significant at the baseline $(p<0.05)$ but this difference was not significant at 6 months after treatment $(p>0.05)$.

\section{Discussion}

This study aimed to assess the gingival health of permanent first molars before and 6 months after restoration with SSC. Gingival parameters are among the most important factors to be evaluated after placement of SSC because SSCs are prefabricated crowns and may not ideally adapt to the tooth surface. In case of defect and misfit, cleaning of restoration margins would be difficult and plaque accumulation and subsequent gingival inflammation may pursue.

Our results showed that in 23 teeth treated with SSCs, PPD in the mesiobuccal and mesiolingual areas significantly decreased 6 months after treatment, which could be attributed to placement of SSC and the children's ability to better clean the mesial surface. The presence of SSC may prevent an increase in PPD because hypoplastic or carious teeth have porosities on their surfaces, which increase the accumulation of pellicles; whereas SSC s have a smooth, polished surface and, thus, decrease the accumulation of pellicle. Also, children are provided with oral hygiene instructions after treatment. In our study, 6-12-year-old children were evaluated (mostly 10-year olds) and they had good cooperation during placement of SSCs and were mostly capable to maintain a good oral hygiene, which further attributed to the improvement in their gingival status. No significant difference was noted in PPD 6 months after placement of SSC in distobuccal and distolingual areas, which may be due to the inability of the child to clean distal surfaces of molar teeth due to no gingival shrinkage in this area. However, the increase in PPD in the distal surface of test teeth was smaller than that in control teeth. The PPD at the mesial surface of control teeth decreased but it remained constant or increased at the distal surface, which further highlights the significance of oral hygiene and its positive effect on gingival parameters in the mesial surface.

Severely damaged teeth often undergo SSC restoration in children. These teeth are often painful (before treatment) and the child may not chew with the painful side and may not properly tooth brush the area. This lack of function and compromised oral hygiene in the area may cause inflammation and subsequent deterioration of gingival parameters.

At 6 months after placement of SSCs, BOP also decreased significantly; the same reduction was also noted in the control group, which may be attributed to the positive effect of oral hygiene instructions given to parents and children and consequent improvement in oral hygiene status, which probably resulted in a reduction of inflammation and BOP. Gingival color also improved due to a reduction in inflammation and BOP.

In general, the use of a well-fitted SSC in permanent first molars associated with oral hygiene instruction can decrease inflammation and improve gingival parameters in children.

A search of the literature yielded no previous study on SSCs for permanent teeth to compare our results with. Gulmann and Matssin evaluated the effect of SSC of primary second molars on periodontal health of adjacent permanent first molars and found no significant difference in gingival index, plaque index, or PPD of mesial surface of permanent first molar and control area. They concluded that a suitable SSC placed on a primary second molar has no adverse effect on periodontal health of adjacent permanent first molar, ${ }^{6}$ which was in agreement with our results.

Sharaf and Farsi found that interproximal bone loss had no significant association with the extension of crown margins, their adaptation, or duration of presence of crown in the oral cavity, while oral hygiene index of children significantly affected their gingival index. They stated that in patients with good oral hygiene, SSC is a suitable modality with no adverse effects on the gingiva ${ }^{9}$. Their results were in agreement with ours since they reported that oral hygiene index significantly affected PPD and BOP. Our study showed that improved oral hygiene decreased gingival inflammation in control teeth that did not undergo any restorative procedure.

Durr and Ashrafi reported that the degree of gingival inflammation was much higher in teeth restored with ill-fitted SSCs compared to other areas, while gingival status around teeth restored with ideal SSCs was similar to that in control areas. ${ }^{4}$ Einwag showed that well-fitted crowns of primary molars were clinically acceptable and caused insignificant gingival irritation; ${ }^{5}$ their results were in accord with ours. In our study, SSCs were placed by pediatric dentists and were compatible with teeth in terms of size. Chao and Tsai reported that unsuitable contour and bulging of SSCs negatively affected gingival index, while supragingival plaque in these teeth played a less significant role in this regard compared to control teeth. Thus, care must be taken by clinicians to well adapt the restoration margins to tooth surfaces and prevent mechanical defect to preserve gingival health. ${ }^{8}$ Salama and Myers showed that cervical margin misfit and plaque accumulation caused moderate to severe gingivitis around teeth restored with such SSCs. ${ }^{7}$

\section{Conclusion}

The results showed that placement of properly fitted SSCs for the restoration of young molar teeth in children with good oral hygiene had no adverse effect on gingival health. Placement of SSCs on permanent first molars in our study decreased PPD, degree of inflammation, and BOP, and improved gingival color. Thus, these crowns are recommended for restoration of young permanent first molars with extensive caries.

\section{ACKNOWLedgment}

The authors would like to thank the Research Deputy of Tehran University of Medical Sciences for supporting this study and 
appreciate the participation of children and cooperation of their parents.

\section{References}

1. McDonald RE, Avery DR. Dentistry for the child and adolescent, 10th ed. St. Louis: Missouri; 2016. pp. 197-198.

2. Randall RC. Preformed metal crowns for primary and permanent molar teeth: review of the literature. Pediatr Dent 2002 Sep-Oct;24(5):489-500.

3. Carranza F, Newman M, et al. Clinical periodontology. Philadelphia: W. B. Saunders Co; 2012.

4. Durr DP, Ashrafi MH, et al. A study of plaque accumulation and gingival health surrounding stainless steel crowns. ASDC J Dent Child 1982 Sep-Oct;49(5):343-346.

5. Einwag J. Effect of entirely preformed stainless steel crowns on periodontal health in primary, mixed dentitions. ASDC J Dent Child 1984 Sep-Oct;51(5):356-359.
6. Guelmann M, Matsson L, et al. Periodontal health at first permanent molars adjacent to primary molar stainless steel crowns. J Clin Periodontol 1988 Oct;15(9):531-533. DOI: 10.1111/j.1600-051X.1988. tb02124.x.

7. Salama F, Myers D. Stainless Steel Crown in clinical pedodontics: a review. Saudi Dent J 1992;4(2):70-74.

8. Chao DD, Tsai TP, et al. Clinical evaluation of gingival tissue restored with stainless steel crown. Changgeng Yi Xue Za Zhi 1992 Dec;15(4):198-203.

9. Sharaf AA, Farsi NM. A clinical and radiographic evaluation of stainless steel crowns for primary molars. J Dent 2004 Jan;32(1):27-33. DOI: 10.1016/S0300-5712(03)00136-2.

10. MacLean JK, Champagne CE, et al. Clinical outcomes for primary anterior teeth treated with preveneered stainless steel crowns. Pediatr Dent 2007 Sep-Oct;29(5):377-381.

11. Roberts C, Lee JY, et al. Clinical evaluation of and parental satisfaction with resin-faced stainless steel crowns. Pediatr Dent 2001 Jan-Feb; 23(1):28-31. 